\section{A Substitute for the Feulgen Staining Technique}

\author{
H. B. W. GREIG \\ From the Department of Haematology, South African \\ Institute for Medical Research, Johannesburg; \\ South Africa
}

(RECEIVED FOR PUBLICATION JUNE 31, 1958)

The Feulgen "nucleal" reaction as applied cytologically (Feulgen and Rossenbeck, 1924) is based on Feulgen's finding that among the products of mild acid hydrolysis of nucleoprotein is a substance which gives a positive Schiff's test (recoloration of leucobasic fuchsin) for aldehydes. The Feulgen reaction is probably specific for deoxyribonucleoprotein (Pearse, 1953 ; Kurnick, 1955); thus nuclear chromatin and chromosomes are stained. Nucleoli and cytoplasm, which contain ribonucleoprotein, are Feulgen negative.

The use of the Feulgen stain in haematological material has been described by La Cour (1944), Pittaluga and Bessis (1944), Gardikas and Israëls (1948), Rheingold and Wislocki (1948), Thoma (1950), and Marinone (1951). The method has been used principally for the localization of deoxyribonucleoprotein and in the study of chromosomes and the processes of cell mitosis. Besides these rather academic uses, the stain has a practical use, that of assisting in the identification of primitive cells by virtue of the clear demonstration of the nucleoli (Hayhoe and Whitby, 1955).

Some years ago, when the author first used the Feulgen stain, he attempted to counterstain blood and bone marrow films with Leishman's stain, but found that stain diluted in the ordinary way with buffered distilled water approximately 1 in 1 to 1 in 2, with the customary staining time of five to 10 minutes, gave useless results on account of the intensity of uptake of the Romanowsky stain by the nuclear chromatin. It was necessary to dilute the Leishman stain about 1 in 10 to 1 in 20 , and to reduce the staining period to about one minute. This resulted in the Feulgenpositive material taking up a deep basophilic colour, while the cytoplasm and nucleoli remained unstained.

The Feulgen staining technique is a fickle one. Peter Gray (1954) says of the Feulgen reaction: "Its nuclear reactions appear to depend upon the selective staining of the thymonucleic acid, but the stain itself is so difficult and so temperamental that it is doubtful that it has occasioned any real advance in microtomy."

Schiff's reagent does not keep well (Lhotka and Davenport, 1949 ; Lillie, 1951). The staining of the nuclei of blood cells is often faint and detail difficult to discern. On account of the improvement in contrast and definition of the Feulgen positive material, the author continued to use diluted Leishman's or Wright's stain after Feulgen staining.

Subsequently, the effect of diluted Wright's or Leishman's stain was tested on smears fixed in the usual way and hydrolysed for 15 minutes at $56^{\circ} \mathrm{C}$. in normal hydrochloric acid omitting the treatment with Schiff's reagent. It was found that this gave similar results to those obtained with diluted Wright's or Leishman's stain after using Schiff's reagent. The technique is easy and the reagents used are immediately available in any haematological laboratory.

\section{Method}

Air-dried smears of blood or bone marrow are fixed for three minutes in absolute methanol, or, better, in absolute methanol containing $10 \%$ formalin (4\% formaldehyde solution). After fixation, the slides are rinsed well with distilled water and then immersed in normal hydrochloric acid at a temperature of $56^{\circ} \mathrm{C}$. and left for 15 minutes. They are rinsed with distilled water and then stained for approximately 30 to 60 seconds with a Romanowsky stain, e.g., Wright's or Leishman's, diluted 1 in 10 with buffered distilled water $(p \mathrm{H} \mathrm{6.8)}$. The slide is then rinsed with distilled water and dried in the air. Fig. 1 shows examples of blood and marrow films stained by this technique. Chromatin and chromosomes are stained a deep blue-black. Nucleoli appear very clearly as "holes" in the nuclei ; cytoplasm is not stained, but azuriphil granules and eosinophilic granules often stain with the eosin component of the Romanowsky stain.

\section{Discussion}

The principal use of this stain (and the Feulgen stain) in diagnostic haematology lies in the demonstration of the presence, size, and number of nucleoli in nuclei. The value of this is greatest in leukaemia, especially in cases of aleukaemic acute leukaemia with "microblasts." These are scanty on the blood films and resemble small lymphocytes when stained by the Romanowsky stains. The use of this stain to demonstrate clearly the presence of nucleoli has been greatly appreciated by technicians.

Although this staining technique appears to stain the same structures as the Feulgen stain does, no claim is made for it as a specific cytochemical procedure for deoxyribonucleoprotein. Further study is required on this point and on the rationale of the procedure.

Flax and Pollister (1949) and Pollister, Himes, and Ornstein (1951) showed that the dye azure A stained the nucleoproteins by virtue of their phosphoric acid groups, but Pollister did not use azure $A$ after acid hydrolysis to differentiate deoxyribonucleoprotein from ribonucleoprotein as the technique described appears to do. The mild acid hydrolysis probably "uncovers" the phosphoric acid groups of deoxyribonucleoprotein, while differentiation probably depends on the greater 


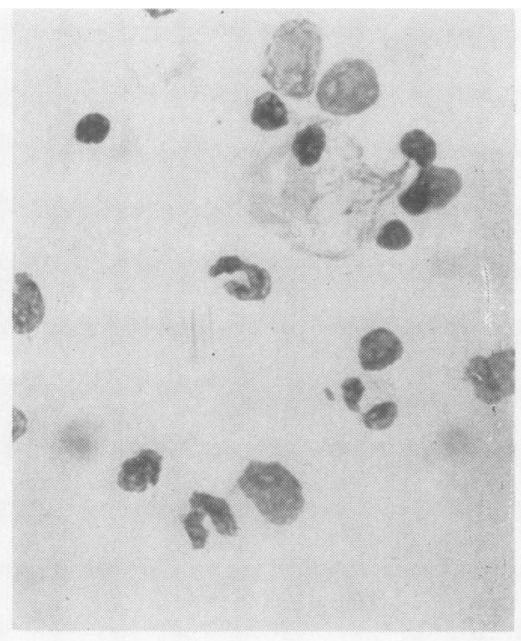

(a)

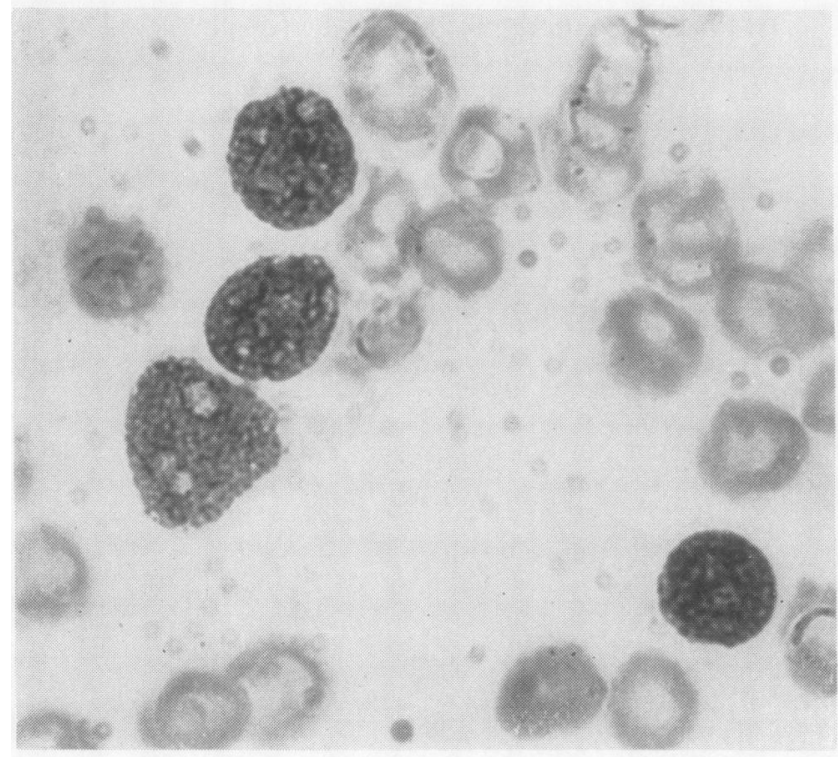

(c)

FIG. 1.- (a) A low-power $(\times 480)$ view of the bone marrow from a case of very early acute leukaemia, showing a marked increase in the number of "blast" colls although normal cells of both the myeloid and erythroid series are present. The peripheral blood showed a leucopenia. (b) A high-power view $(\times 1,200)$ of two of the "blasts" from the same marrow. (c) and $(d)$ " Blasts" in blood films of two different cases of acute leukaemia $(\times 1,200)$.

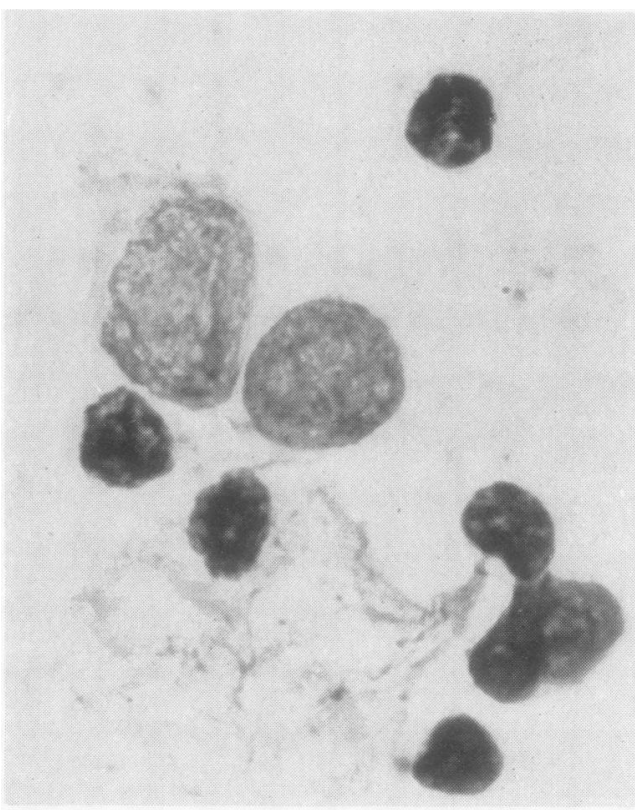

(b)

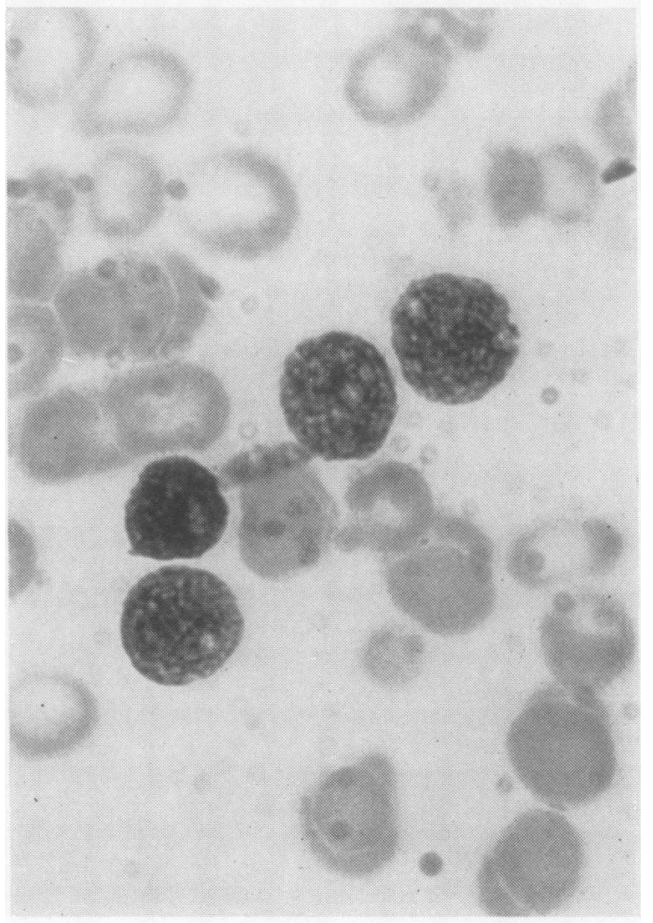

(d) 
sensitivity of ribonucleic acid to acid hydrolysis (Kurnick, 1955) and the fact that ribonucleic acid is very easily removed from the cell by such treatment (Vendrely-Randavel, 1949).

\section{REFERENCES}

Feulgen, R., and Rossenbeck, H. (1924). Hoppe-Seylers Z. physiol. Chem., 135, 203.

Flax, M., and Pollister, A. W. (1949). Anat. Rec., 105, 536.

Gardikas, C., and Israëls, M. C. G. (1948). J. clin. Path., 1, 226.

Gray, P. (1954). The Microtomist's Formulary and Guide. Blakiston New York. Constable, London.

Hayhoe, F. G. J., and Whitby, L. H. (1955). Brit. J. Haemat., 1, 1.

Kurnick, N. B. (1955). Int. Rev. Cytol., 4, 221.

La Cour, L. F. (1944). Proc. roy. Soc. Edinb., B, 62, 73.

Lhotka, J. F., and Davenport, H. A. (1949). Stain Technol., $24,237$.

Lillie, R. D. (1951). Ibid., 26, 163.

Marinone, G. (1951). Rev. belge Path., $21,333$.

Pearse, A. G. E. (1953). Histochemistry. Churchill, London.

Pittaluga, G., and Bessis, M. (1944). Bull. Histol. Techn. micr., 21, 23. Pollister, A. W., Himes, M., and Ornstein, L. (1951). Fed. Proc., $10,629$.

Rheingold, J. J., and Wislocki, G. B. (1948). Blood, 3, 641.

Thema, K. (1950). Schweiz. med. Wschr., 80, 145.

Vendrely-Randavel, C. (1949). C.R. Soc. Biol. (Paris), 143, 294.

\section{The "Lambeth" Technitray}

\section{O. A. N. HUSAIN}

From the Group Laboratory, Lambeth Hospital, London

\section{(RECEIVED FOR PUBLICATION DECEMBER 9, 1958}

The biggest obstacle to the design of an all-purpose technician's carrying unit has always been that of the widely differing requirements of individual laboratories. An attempt to overcome it has been made by the construction of a basic tray with a drawer beneath and an extensive range of interchangeable inserts.

The final design is a compromise between the numerous ideas from this and other laboratories and the limiting factors of construction and costs.

Made throughout of thin, polished aluminium, the whole unit, with the standard set of components as illustrated (Fig. 1), measures $14 \times 8 \times 5$ in. with the handle 10 in. high, and weighs under $4 \mathrm{lb}$. Being metal, it is easily cleaned and sterilized and a separate slip-on plastic cover allows it to be carried in inclement weather.

The drawer below, 2 in. deep, can be used for syringes or notes, or be fitted with further inserts. The tray, as shown, provides for 24 red or white blood cell bulb type pipettes (in two layers), 18 bijou bottles, 22 Kahn tubes, five "universal" containers,

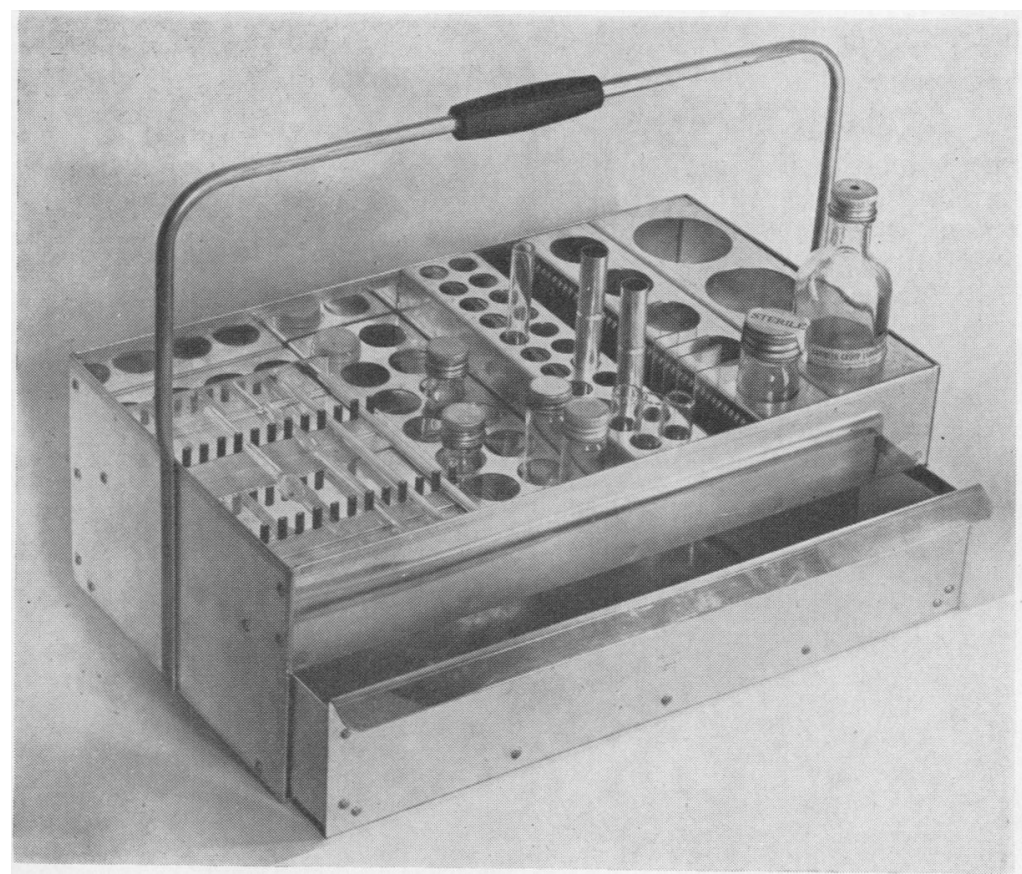

Fig. 1.-The Lambeth "technitray." 